\title{
Using Interactive Fiction to Teach Clinical Decision-Making in a PharmD Curriculum
}

\author{
Noam Morningstar-Kywi ${ }^{1}$ (1) $\cdot$ Rory E. Kim ${ }^{1}$ (1)
}

Accepted: 5 February 2021 / Published online: 23 February 2021

(c) International Association of Medical Science Educators 2021

\begin{abstract}
Teaching of clinical decision-making is an important component of health professions education. Patient case examples are widely used in didactic coursework to teach this material, but engaging all learners in large, lecture-based courses remains a challenge. Interactive fiction (IF), a digital choose-your-own-adventure media, provides an accessible way for students to individually explore the narrative of a patient-case in a safe environment. Here, we report the development of interactive, digital patient-cases (eCases) using Twine, a free IF development platform. Fourteen eCases were developed in collaboration with 11 faculty members and were used in seven different PharmD courses over three semesters. eCase content was developed by faculty members for their respective instructional topics and accessed via Web browsers on students' personal electronic devices. eCases were received positively by students, with $>90 \%$ of students reporting that eCases were easy to use, helped them learn the material at their own pace, and gave them an opportunity to learn from mistakes. Student self-perceived confidence also increased significantly after eCase use. Faculty reported that eCases took more time to develop than conventional cases, but were easier to deliver and provided better student engagement. IF is an accessible media for creating and delivering low-fidelity interactive patient cases that can engage all students in a large class. eCases allow students to apply their knowledge, practice clinical decision-making, and safely learn from their mistakes. eCases are versatile and well suited for both in-person and virtual teaching across a variety of health professions programs to teach clinical decision-making.
\end{abstract}

Keywords Educational technology $\cdot$ Simulation · Clinical decision-making · Pharmacy education · Interactive fiction · Active learning

\section{Introduction}

Healthcare professions graduates must perform sound clinical reasoning and solve therapeutic problems in high stakes environments. In medical and pharmacy school curricula, these skills are often first introduced in preclinical courses using patient cases, prior to engagement with real patients during experiential learning [1-3]. Delivery of case-based sessions in a large classroom generally includes guiding students through the details of a patient case from presentation to treatment [1]. This may be augmented by active learning strategies, including audience response systems, Socratic

Rory E. Kim

Rocallag@usc.edu

1 Department of Clinical Pharmacy, School of Pharmacy, University of Southern California, 1985 Zonal Ave, Los Angeles, CA 90089, USA questioning, role play, and games [4-10]. According to Knowles' principles of adult learning, learning should be problem-centered, have immediate relevance to the learner, emphasize experience including making mistakes, and include learners in planning and evaluation [11]. Conventional case delivery is problem-centered, but the majority of the literature to support case-based learning is in the context of small group sessions rather than large classroom lectures. With larger class sizes, it is difficult to engage multiple learners at different levels and for instructors to provide prompt, individualized feedback to correct mistakes [8, 12].

High fidelity application-based simulations and assessments, such as virtual patients (VPs), objective structured clinical examinations (OSCEs), and interaction with standardized patients, engage learners individually, and provide deep learning and patient application in a safe environment [13-15]. VPs have been defined as "a specific type of computer program that simulates real-life clinical scenarios; learners emulate the roles 
of health care providers to obtain a history, conduct a physical exam, and make diagnostic and therapeutic decisions" $[16,17]$. This is accomplished without the risk of harming a real patient [18]. In a review of VPs for medical education, it was noted that they are best suited for teaching clinical reasoning [17]. These types of programs may also provide student performance data to faculty to allow them to formatively assess students and target further instruction [19]. However, VPs require significant financial resources and time to develop and deploy [16-18]. Thus, we identified a need for a method of case delivery that is accessible, individualized, interactive, and cost-effective, permitting use in didactic sessions while adhering to Knowles' principles of adult learning.

To meet these needs, we developed interactive, electronic patient cases (eCases) using Twine, a free, open-source tool for creating interactive fiction (IF) [20]. IF is a digital media for delivering computer-based "choose-your-own-adventure" stories. IF was first used in the 1970s with the advent of personal computing [21] and is typically used to develop textbased adventure games, where players take on the role of a character to explore the outcome of a series of decisions [22, 23]. The underlying principles of IF are the same as printed "choose-your-own-adventure" books: choices made by the reader lead to different results, different subsequent choices, and different outcomes, thus moving the narrative towards a final end result [23]. When leveraged for learning in health science education, this process parallels clinical decisionmaking. Different learners select different tests to order and medication(s) to prescribe, and these choices lead to different results, different choices at follow-up, and ultimately different patient outcomes. The digital nature of IF grants the ability to track, store, and adjust variables (e.g., medication dose, lab values, time, number of adverse events, questions asked, etc.), the potential to make multimodal choices (e.g., multidrug therapeutic regimens at varying doses), the capability to collect, assess, and report user progress in real time, and the potential to scale distribution to a class of any size. To our knowledge, there are no currently published reports on the use of IF in pharmacy or medical education. The purpose of this study is to describe the development, implementation, and student and faculty response to eCases using IF in a PharmD curriculum.

\section{Methods}

\section{eCase Development}

We developed eCases using Twine (twinery.org), an open-source IF development platform [20]. Stories (cases, for our purpose) are written in Twine using its own coding language, which is later handled internally by a JavaScript-based interpreter. Twine then publishes the story to an HTML file containing the content of the story as well as the JavaScript engine. Native HTML and
JavaScript functions are embeddable in the story, allowing for additional capabilities, such as the display of Web-linked content or the export of data to a remote server.

The content for each eCase was developed by individual faculty members, generally derived from a paper-case or patient case example they were planning to use for a scheduled classroom session on a given topic or disease state. Student workers were employed to code the content of the case into Twine. Broadly, faculty members provided a patient case scenario (e.g., clinical pharmacist consulting on a patient in the cardiology ward), augmented by supporting data such as a patient history and lab values. Additionally, a set of information-gathering questions and answers, a list of interventions to choose from, and a series of outcomes for each intervention or combination of interventions was provided. After initial coding of this material, each eCase underwent a collaborative process of further development, testing, and debugging prior to deployment.

Development required 2 to $4 \mathrm{~h}$ of faculty time in addition to usual time allotted for conventional case development, and 6 to $10 \mathrm{~h}$ of student worker time, scaling with the complexity of the case. The majority of this work was done individually, with each eCase requiring one to two hours of in-person or virtual meetings between the faculty member and student worker.

\section{eCase Implementation}

eCase HTML files published by Twine were uploaded to learning management software (Blackboard), where they could be accessed by students on course pages and executed locally by a web browser on the students' personal electronic devices. User progress data was collected and stored on a server managed by the school; personally identifiable information was not collected except where specified and with the explicit consent of the students.

eCases were made available to the students at the time deemed appropriate by the instructor, and generally were left available until the end of the course. Course implementation was typically structured such that after each eCase was introduced, students would have 10-15 min to complete the eCase one or more times, followed by an in-class debrief in most instances. In situations where eCases were used as an at-home activity or for a virtual class (due to COVID-19), a debrief opportunity was scheduled for a later time.

\section{Assessment of eCase Implementation}

Data were collected through separate surveys to assess student and faculty satisfaction and perceptions of eCases. Surveys assessing student experiences were distributed via Qualtrics after completion of the eCase and contained standardized questions used for all eCases along with up to two topicspecific questions. The standardized questions assessed 
accessibility, utilization, and perceived benefits. Student responses on the standardized question set were analyzed using descriptive statistics. Topic-specific questions were included at the discretion of, and written by, the developing faculty member. A "before and after" format assessing selfperceived changes in confidence was recommended for these questions, but the ultimate verbiage was left to the faculty member. Where possible, changes in self-perceived student confidence from pre- to post-eCase use were assessed using the Wilcoxon signed rank test. An a priori alpha level of 0.05 was used to determine statistical significance. Statistical analyses were performed using Microsoft Excel.

Faculty experiences were collected at the end of each semester using a single Qualtrics survey. The faculty survey focused on differences in development and implementation between eCases and "usual" or conventional patientcases, defined as the way in which that faculty member had delivered patient cases in classroom sessions prior to using an eCase. Descriptive statistics were used to characterize faculty responses. All surveys distributed to both students and faculty also contained free response fields to collect qualitative feedback. This study was reviewed and deemed exempt by our institutional review board.

\section{Results}

\section{eCase Use}

At present, 14 eCases, developed in collaboration with 11 faculty, have been used in seven different PharmD courses over 3 semesters, as well as in one international program course and one continuing education course. Five of these eCases have been used for multiple course offerings. A full breakdown of topics and courses is given in Table 1. Two eCases were used in an exclusively virtual setting after courses were moved online in response to COVID19.

\section{eCase Development: Content and Features}

The use of a codable interactive fiction platform allows for flexibility in the customization of each eCase. The general flow of eCases is depicted in Fig. 1. The following section notes several common features to describe functionality of eCases and the utilization of IF as a teaching tool. Not all eCases included all features described below, but these are representative of the capabilities and flow. An example eCase, focused on recommending proper over the counter medication regimens for cough, cold, and flu symptoms, is available online (http://psp.acadoinformatics.com/DemoeCase). Screenshots from this and other eCases are referred to in the following text and provided in Supplementary Fig. 1.

Upon opening the eCase, students are presented with an introductory passage describing the scenario and presenting the patient (Supplementary Fig. 1A). Students then have an opportunity to gather more information through one or more of the following: exploring a mock EMR built into the eCase (Supplementary Fig. 1B), reading an inserted provider note (Supplementary Fig. 1C), ordering tests or labs (Supplementary Fig. 1D), or asking the patient or patient's agent questions from a menu of options

Table 1 eCases used in PharmD courses and other settings

\begin{tabular}{|c|c|c|}
\hline Topic & Course & Uses \\
\hline Acute heart failure & Therapeutics: Cardiovascular System (P3) & 1 \\
\hline Aminoglycoside dosing & Pharmacokinetics (P2) & 1 \\
\hline Community-acquired pneumonia & Therapeutics: Infectious Diseases (P3) & 1 \\
\hline Cough, cold, and flu & Non-Prescription Therapies (P1) & 1 \\
\hline Diabetic ketoacidosis and hyperglycemic hyperosmolar syndrome & Therapeutics: Endocrine System (P2) & $1^{\mathrm{a}}$ \\
\hline Malaria chemoprophylaxis & Community Pharmacy Practice (P2/P3) & 2 \\
\hline Pharmacogenetics of acute coronary syndrome & PGxLEARN (CE) & 1 \\
\hline Rheumatoid arthritis & $\begin{array}{l}\text { Therapeutics: General Medicine and Inflammatory } \\
\text { Disorders (P2) }\end{array}$ & 1 \\
\hline Self-administered hormonal contraception & Community Pharmacy Practice (P2/P3) & 2 \\
\hline Smoking cessation (state protocol) & Community Pharmacy Practice (P2/P3) & 2 \\
\hline Smoking cessation (collaborative practice agreement) & $\begin{array}{l}\text { Non-Prescription Therapies (P1) } \\
\text { International Student Summer Program (UG) }\end{array}$ & $3^{\mathrm{a}}$ \\
\hline Travel medicine & Community Pharmacy Practice (P2/P3) & 2 \\
\hline Vancomycin dosing & Pharmacokinetics (P2) & 1 \\
\hline Warfarin dosing & Therapeutics: Cardiovascular System (P3) & 1 \\
\hline
\end{tabular}

$P 1$ pharmacy year 1, $P 2$ pharmacy year 2, $P 3$ pharmacy year 3, $U G$ undergraduate, $C E$ continuing education

${ }^{a}$ Includes use in virtual classroom sessions during COVD19 pandemic 
Fig. 1 Simplified diagram depicting the branching decision tree and multiple pathways for completing an eCase. Outcomes $B$ and D represent the result of optimal choices, whereas as outcomes $\mathrm{A}$ and $\mathrm{C}$ result from suboptimal and inappropriate choices, respectively. In practice, the number of drug choices, iterations, and outcomes can be increased ad infinitum

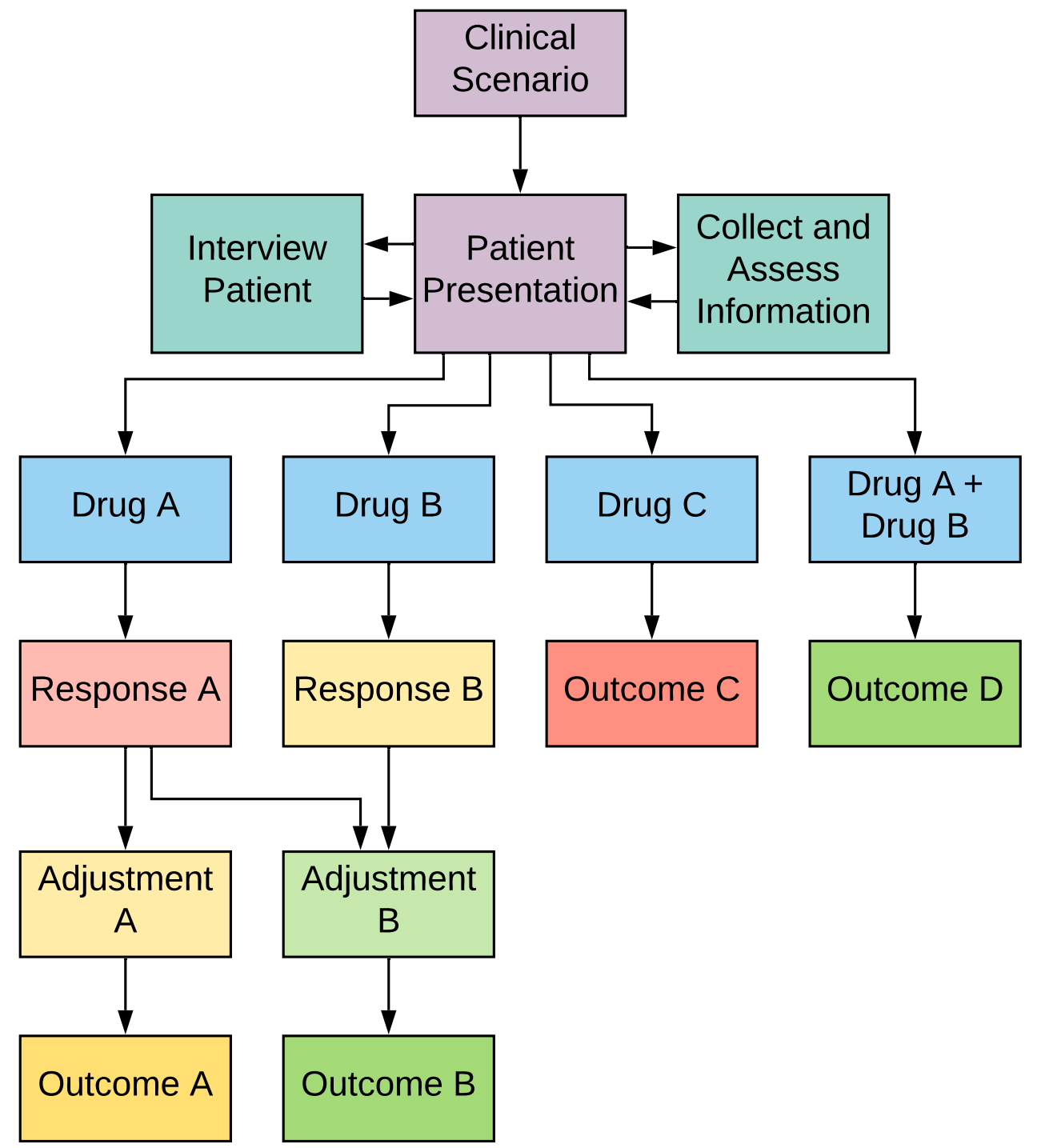

(Supplementary Fig. 1E). It is possible to randomize some or all of this information within a predefined range.

The eCase can be setup such that when certain questions are asked, additional follow-up questions become available (Supplementary Fig. 1F). It is also possible to limit the total number of questions a student can "ask" from the menu before they "run out of time" (Supplementary Fig. $1 \mathrm{G})$, requiring the student to prioritize the most important questions. This principle also applies to ordering tests (e.g. "The patient's insurance will not cover additional tests").

After collecting this information, students are expected to make an assessment of the patient. A learning checkpoint can be inserted at this point (Supplementary Fig. 1H), with the results giving immediate feedback to the student, or being relayed electronically to the instructor for a formative assessment of the class's understanding. Generally, students are allowed to proceed through an incorrect initial assessment without being corrected, allowing them to see how the scenario will play out and learn from their mistakes (the eCase and/or instructor will give feedback at the end).

Next, students select a treatment regimen (pharmacologic and/or non-pharmacologic interventions) for the patient. This can be as simple as selecting a single drug from a menu (Supplementary Fig. 1I), or as complex as combining multiple medications from a menu of choices into a regimen, with the option to select dose, route, frequency, and duration (Supplementary Fig. 1J). Upon proceeding, the eCase calculates the initial outcome of the student's choices, based on criteria defined by faculty during development. For student choices that do not lead to immediate success or failure, students are provided information about the patient response to the selected treatment (Supplementary Fig. 1K). This information can be entirely predefined (e.g., if an ACEinhibitor is chosen, patient presents at the next visit with cough) or calculated mathematically (e.g., for every unit of 
insulin administered, decrease BG by $30 \mathrm{mg} / \mathrm{dL}$ ). Students then make any necessary changes to the treatment regimen, adjusting for side effects or lack of efficacy and rectifying any mistakes.

Upon completion of the case, whether from successful management of the patient, failing to treat the patient successfully in time, or selecting options that harmed the patient, the students are presented with feedback (Supplementary Fig. 1L, M). This feedback is customized based on their choices and outcome. Students are then given an opportunity to replay the eCase, exploring new options or adapting their approach in response to previous outcomes. A key feature of eCases is the ability to include multiple pathways to both success and failure.

The number of times students can adjust therapy or respond to adverse events varies by eCase. Certain topics, including diabetic ketoacidosis/hyperglycemic hyperosmolar syndrome, warfarin dosing, and aminoglycoside and vancomycin dosing, focus on titrating the dose of a medication to achieve a specific concentration or biomarker range (determined by developing faculty). Other topics, such as rheumatoid arthritis and community-acquired pneumonia, focused more on selection of appropriate agents rather than dose. The most complex eCases, such as acute heart failure, required students to both identify a combination of suitable agents and select the appropriate dose, route, and frequency.

\section{User Progress Data}

Four faculty elected to set up five eCases to collect anonymous data on user (student) choices. Upon user completion of the eCase, a data packet containing choices and outcome(s) was sent to a spreadsheet accessible to the faculty member. In cases where patient information was randomized, the initial condition was also included. Because the spreadsheet updated in real-time, faculty were able to use this information for in-class formative assessment, adjusting their teaching or debrief accordingly. Supplementary Table 1 contains an example of a user data collection spreadsheet, with column headers edited for readability; the full spreadsheet for the example eCase is also available online for viewing (http://psp.acadoinformatics.com/eCase-UserProgress-Report).

\section{Student Feedback}

Response rates for the student survey issued after each eCase are also shown in Table 2. Not all students within the class completed each eCase, and not all students completing the eCase responded to the respective surveys. At the time of survey administration, successful completion of the eCase ranged from 63 to $100 \%$ of respondents. Median number of attempts before reaching a successful outcome ranged from two to eight, scaling with eCase difficulty and correlating with the overall success rate. The number of students that reported replaying the eCase after reaching a successful outcome ranged from 50 to $89 \%$. Results for the standardized utility questions were pooled between all cases and are shown in Table 3. Although the number of respondents varied widely between cases, trends were consistent across all eCases and match the results shown in the table.

The topic specific questions for the three most recent eCases used a consistent format. Each inquired about students' reported confidence in both patient assessment
Table 2 PharmD student survey response rates

\begin{tabular}{lll}
\hline Topic & Responses $^{\mathrm{a}}$ & Class size $^{\mathrm{b}}$ \\
\hline Acute heart failure & 8 & 185 \\
Aminoglycoside dosing & 17 & 196 \\
Community-acquired pneumonia & 16 & 187 \\
Cough, cold, and flu & 179 & 193 \\
Diabetic ketoacidosis and hyperglycemic hyperosmolar syndrome & 21 & 199 \\
Malaria chemoprophylaxis & 22 & $100^{\mathrm{c}}$ \\
Rheumatoid arthritis & 24 & 195 \\
Self-administered hormonal contraception & 22 & $100^{\mathrm{c}}$ \\
Smoking cessation (state protocol) & 22 & $100^{\mathrm{c}}$ \\
Smoking cessation (collaborative practice agreement) & 153 & $391^{\mathrm{c}}$ \\
Travel medicine & 22 & $100^{\mathrm{c}}$ \\
Vancomycin dosing & 17 & 196 \\
Warfarin dosing & 19 & 185 \\
Total & 542 & \\
\hline
\end{tabular}

${ }^{a}$ eCase and survey completion were not mandatory in all courses. Includes partial responses

${ }^{\mathrm{b}}$ Reflects enrollment in course and course-year (Table 1) at time of eCase administration

${ }^{\mathrm{c}}$ For eCases used multiple times, class size is the sum of all classes 
Table 3 Pooled student responses

\begin{tabular}{lllllll}
\hline Question: This eCase... & SD & D & N & A & SA & Total \\
\hline Was easy to use & 13 & 12 & 16 & 140 & 225 & $406^{\mathrm{a}}$ \\
Helped me to learn the material & 11 & 2 & 30 & 132 & 323 & 498 \\
$\begin{array}{l}\text { Helped me to check my understanding of the material } \\
\text { Increased my confidence in applying my knowledge of }\end{array}$ & 9 & 4 & 26 & 122 & 338 & 499 \\
$\quad$ the material & & 17 & 39 & 123 & 216 & $405^{\text {a }}$ \\
$\begin{array}{l}\text { Allowed me to learn at my own pace } \\
\text { Gave me an opportunity to learn from mistakes }\end{array}$ & 9 & 7 & 25 & 132 & 325 & 498 \\
\hline
\end{tabular}

Data are shown as the number of students in each category

$S D$ strongly disagree, $D$ disagree, $N$ neither agree nor disagree, $A$ agree, $S A$ strongly agree

${ }^{a}$ This question was not included on the survey disseminated with the first eCase deployed and treatment planning. Results comparing the confidence of students in those areas before and after each eCase are shown in Table 4. The Wilcoxon signed rank test showed that the students' confidence increased significantly after the use of the eCases. The remaining topic specific questions and responses are given in Supplementary Table 2.

\section{Faculty Feedback}

Of the 11 faculty who worked to develop an eCase, nine fully completed the faculty feedback survey. Compared to their usual patient cases, six faculty indicated that it took moderately or significantly more time to develop an eCase, while the remainder reported eCases took a similar or lesser amount of time to develop. The eCase development process was unanimously rated as easy to follow, efficient, and reasonably timed. Table 5 shows the perceptions of faculty regarding the time and effort required for delivery of eCases, as well as perceived utility. Faculty indicated that allotting 15-20 min for eCase completion and debrief was sufficient in most instances. All faculty reported that they used the formative feedback provided to address gaps in knowledge and misconceptions, and to build to more complex topics. All faculty developers indicated they would be interested in developing additional eCases at the time of the survey response; 3 have done so to-date.

\section{Accessibility}

Students reported accessing eCases on a variety of devices, including laptops, iPads, other tablets, and smartphones. Over the last 3 semesters, only one student reported difficulty accessing and using an eCase. This occurred during the first eCase deployment and was attributed to the student not having a personal electronic device with them in class. No faculty reported difficulty in uploading the eCase file to Blackboard, accessing it at the appropriate time, or accessing user data spreadsheets.
Table 4 Changes in student confidence

\begin{tabular}{llll}
\hline & $\begin{array}{l}\text { Before completing the } \\
\text { eCase }\end{array}$ & $\begin{array}{l}\text { After completing the } \\
\text { eCase }\end{array}$ & $P$ value \\
\hline $\begin{array}{l}\text { Smoking cessation (collaborative practice } \\
\text { agreement) }\end{array}$ & & & \\
$\quad \begin{array}{l}\text { Make an appropriate assessment } \\
\text { Recommend treatment }\end{array}$ & $3(1)$ & $4(0)$ & $P<0.00001$ \\
$\begin{array}{l}\text { Cough, cold, and flu } \\
\quad \text { Make an appropriate assessment }\end{array}$ & $3(2)$ & $4(0)$ & $P<0.00001$ \\
$\quad$ Recommend treatment & $3(1)$ & $4(1)$ & $P<0.00001$ \\
$\begin{array}{l}\text { Diabetic ketoacidosis and hyperglycemic } \\
\text { hyperosmolar syndrome }\end{array}$ & & $4(1)$ & $P<0.00001$ \\
$\quad \begin{array}{l}\text { Make an appropriate assessment } \\
\text { Recommend treatment }\end{array}$ & $3(3)$ & $4(1)$ & $P=0.00188$ \\
\hline
\end{tabular}

All questions were in the form of a Likert-like scale evaluation from $1=$ not confident to $5=$ very confident. Results are expressed as a median (interquartile range). Differences in scores before using the eCase and after using the eCase were calculated using a Wilcoxon signed rank test 
Table 5 Faculty perceptions of development and utilization of eCases

\begin{tabular}{llllll}
\hline Relative ${ }^{a}$ outcome & Much less & Somewhat less & $\begin{array}{l}\text { About the same } \\
\text { Some- } \\
\text { what } \\
\text { more }\end{array}$ & Much more \\
\hline Time and effort & & & & & \\
$\quad$ Time to prepare before class & 2 & 2 & 2 & 2 & 1 \\
Effort to prepare before class & 1 & 3 & 1 & 2 & 2 \\
Time to deliver in class & 1 & 1 & 3 & 1 & 0 \\
Effort to deliver in class & 1 & 6 & 2 & 0 & 0 \\
Utility & & & & & \\
Facilitated engagement & 0 & 0 & 0 & 5 & 4 \\
Facilitated discussion & 0 & 0 & 0 & 2 & 5 \\
$\quad$ Provided formative feedback & 0 & 0 & 2 & 2 & 7 \\
$\quad$ Provided real-world application & 0 & 0 & 0 & 2 \\
\hline
\end{tabular}

Data are shown as the number of faculty in each category $(n=9)$

aRelative to 'conventional' case preparation and delivery. Conventional cases were defined as the style of patient cases used by responding faculty prior to using an eCase

\section{Discussion}

Using interactive fiction, we developed a novel patientcase delivery method that adapts to user choices and allows learners to explore the narrative of a clinical case scenario from patient presentation to therapeutic outcome. This approach provides a method for teaching and practicing clinical decision making that is accessible across multiple levels of material and learner capabilities. eCases were well received by students and faculty alike, and were perceived to be useful, affordable, and worthwhile, permitting successful uptake in our PharmD curriculum.

The IF framework is designed for creating narrative games. Thus, the framework is well-suited for bringing elements of gaming, or gamification, into the learning process. Gamification has been shown to enhance learner engagement and introduce fun in both pharmacy and medical education [19, 24, 25]. The IF framework used here leveraged game elements to engage learners in exploring clinical case scenarios [25]. The IF development platform is flexible, allowing eCases to be created for a wide variety of topics, and spanning different levels of complexity for different levels of learners (from the first professional year through continuing education). The general structure of all eCases adhere to the same andragogical principles, but no unified template is required, meaning that the types of user input and choices, outcomes, and feedback can be customized for each topic. The method of deployment is equally flexible, permitting implementation specific to the needs of individual courses or topics. This has become especially apparent with eCases developed and used after classes were moved online in response to the COVID-19 pandemic. As there are free platforms available for creating IF, student-worker or programmer time to code the eCases is the only cost associated with development and deployment.
eCases are similar to virtual patients (VPs) in that they both promote application of clinical knowledge and skills within a digital environment, thus allowing students to practice patient care without risk to actual patients [13, 14, 17, 18, 26-28]. A hallmark of VPs is their use of natural language processing and realistic graphical interfaces to create a user experience that mirrors real-life patient interactions-a feature that is intended for practicing communication and open-ended information gathering [17, 29, 30]. These features have not consistently been shown to be of benefit to learners [17, 29]. In a randomized trial, a menu-driven virtual patient was found to be more helpful for structuring clinical information and more suitable for pre-clinical learners [30]. eCases forego the highfidelity approach in favor of focusing on therapeutic decisionmaking and responding to patient outcomes, which are thought to be primary strengths of teaching with VPs [17,31]. This lowfidelity approach requires significantly less time and resources to develop and deploy. The time to develop a VP case has been reported to take from 22 to over $50 \mathrm{~h}$ to create [14, 32], while eCases took an average of six to $10 \mathrm{~h}$ to develop. Furthermore, the costs to develop VPs has been reported to be $>\$ 10,000$ [27] and may be charged by student (\$75/year) and faculty (\$1100) user [14] resulting in higher costs for larger class sizes and limiting replayability across different groups of learners. In contrast, there are multiple, free IF development platforms, and the only cost to developing an eCase is paying for the coding, which is a one-time expense. eCases can be accessed and replayed by as many users as the faculty author allows. Another key feature of decision-based games and VPs is the ability to track student decision-making. This tracking allows faculty to identify errors in thinking and further target teaching towards learner needs, thus keeping instruction learnercentered [19]. eCases can likewise track student selections and a real-time report is available for instructor use in the live or virtual classroom. While our faculty chose to collect this 
data anonymously, it is feasible to link student choices with an identifier (e.g., student ID number).

Student survey response rates varied depending on the eCase and course, and generally were higher when the instructor provided time to complete the survey in class. Students reported that the eCases were easily accessible, helped them to learn the material, facilitated learning from mistakes, and allowed them to learn at their own pace. The ability to explore multiple patients and outcomes within a single eCase creates a high replay value for students. Though impact on student performance was not directly measured in this analysis, students consistently reported significantly increased confidence in applying knowledge following the utilization of the eCases. The high levels of student satisfaction with eCases compared to traditional case delivery is consistent with that previously reported for other virtual case media [14]. Student feedback remained positive when eCases were used for online classes in Spring 2020.

While eCases can require more faculty time and effort upfront to develop, this is offset by decreased time and effort to deliver, as well as improved learner engagement and application of knowledge. Beyond this initial investment, little or no effort is required to use the same eCase for future course offerings. The ability of eCases to give class-wide formative assessment of student progress was highly valued among faculty. Whereas with large lectures or smaller breakout groups the instructor can only interact with one student or group at a time, the digital feedback provided by eCases allows the instructor to get a snapshot of the entire class's understanding. This has allowed instructors to address misconceptions or gaps in knowledge. It also provides insight into students' approach to clinical decision-making in their pre-clinical years, which has historically been challenging to characterize [33].

eCases are not intended to be a substitute for case conference discussions, higher-level simulation labs, or OSCEs. Rather, they are meant to augment learning prior to any of those activities, allowing students to arrive at their discussion, lab, or summative assessment better prepared for the respective activity. The use of an existing open-source platform has allowed for eCases to be implemented rather quickly and with relatively low expense. Although eCases were positively received by students and faculty, it is important to note that the impact of eCases on student performance was not assessed in this study. Prospective quantitative assessments of the effects of eCases on learning outcomes are planned for future studies.

\section{Conclusion}

Interactive fiction provides an affordable and accessible way to develop and deliver engaging, digital patient cases. The interactive nature, branching narrative, and adaptive capabilities allow students to explore multiple therapeutic pathways and practice clinical decision-making in a safe environment. The flexible nature of eCases allows for easy implementation in lecture-based courses, where they provide an opportunity for active application of knowledge and can give formative feedback to students and instructors. eCases are especially useful in the current environment, where there is a need for active learning tools compatible with online teaching.

Supplementary Information The online version contains supplementary material available at https://doi.org/10.1007/s40670-021-01245-7.

Availability of Data and Material The datasets generated during and/or analyzed during the current study are available from the corresponding author on reasonable request.

\section{Declarations}

Ethics Approval This study was deemed Exempt by the Institutional Review Board (waiver attached).

Conflict of Interest/Competing interests The authors declare that they have no conflicts of interest.

\section{References}

1. Vyas D, Ottis EJ, Caligiuri FJ. Teaching clinical reasoning and problem-solving skills using human patient simulation. Am J Pharm Ed. 2011;75(9):189.

2. Rencic J, Trowbridge RL, Fagan M, et al. Clinical reasoning education at US medical schools: results from a national survey of internal medicine clerkship directors. J Gen Intern. 2017;32:1242-6.

3. Jeffres MN, Kufel WD, Biehle LR, et al. A comprehensive survey of infectious diseases curriculum among United States Pharmacy Schools [published online ahead of print]. Am J Pharm Ed. 2019. Accessed 10 Oct 2020.

4. Prince M. Does active learning work? A review of the research. J Eng Educ. 2004;93(3):223-31.

5. Dominguez-Colman L, Mehta SU, Mansourkhani S, et al. Teaching psychiatric emergencies using simulation: an experience during the boot camp. Med Sci Educ. 2020. https:// doi.org/10.1007/s40670-020-01095-9.

6. Gleason BL, Peeters MJ, Resman-Targoff BH, et al. An activelearning strategies primer for achieving ability-based educational outcomes. Am J Pharm Educ. 2011;75(9):186.

7. McLaughlin JE, Roth MT, Glatt DM, et al. The flipped classroom: a course redesign to foster learning and engagement in a health professions school. Acad Med. 2014;89(2):236-43.

8. Nicholl TA, Lou K. A model for small-group problem-based learning in a large class facilitated by one instructor. Am J Pharm Educ. 2012;76(6):117.

9. Clauson KA, Alkhateeb FM, Singh-Franco D. Concurrent use of an audience-response system at a multi-campus college of pharmacy. Am J Pharm Educ. 2012;76(1):6.

10. Kugler AJ, Gogineni HP, Garavalia LS, et al. Learning outcomes and student preferences with flipped vs lecture/case teaching model in a block curriculum. Am J Pharm Educ. 2019;83(8):7044.

11. Pappas C. The adult learning theory - Andragogy - of Malcolm Knowles. eLearning Industry. May 9, 2013. Accessed 9 Oct 2020. 
12. Duncan-Hewitt WC. A focus on process improves problembased learning outcomes in large classes. Am J Pharm Educ. 1996;60(4):408-16.

13. Berman NB, Durning SJ, Fischer MR, et al. The role for virtual patients in the future of medical education. Acad Med. 2016;91(9):1217-22.

14. Smith MA, Siemianowski LA, Benedict N. Virtual patient case sharing across two schools of pharmacy. Am J Pharm Educ. 2016;80(9):153.

15. Ericsson KA. Acquisition and maintenance of medical expertise: a perspective from the expert performance approach with deliberate practice. Acad Med. 2015;90(11):1471-86.

16. Effective use of educational technology in medical education. Summary report of the 2006 AAMC Colloquium on educational technology. Washington DC: Association of American Medical Colleges; 2007.

17. Cook DA, Triola MM. Virtual patients: a critical literature review and proposed next steps. Med Educ. 2009;43:303-11.

18. Jabbur-Lopes MO, Mesquita AR, Silva LA. Virtual patients in pharmacy education. Am J Pharm Educ. 2012;76(5):91.

19. McCoy L, Lewis JH, Dalton D. Gamification and multimedia for medical education: a landscape review. J Am Osteopath Assoc. 2016;116(1):22-34.

20. Twine. Interactive Fiction Technology Foundation. Updated October 7, 2019. Accessed 10 Sept 2020. https://twinery.org.

21. Jerz DG. Somewhere nearby is colossal cave: examining Will Crowther's original "adventure" in code and in Kentucky. Digital Humanities Quarterly. 2007;1(2). http://www.digitalhumanities. org/dhq/vol/1/2/000009/000009.html.

22. Rieber LP. Computers, graphics, and learning. Madison: Brown \& Benchmark; 1994.

23. Kozdras D, Haunstetter DM, King JR. Interactive fiction: 'new literacy' learning opportunities for children. E-Learning. 2006;3(4):519-33.
24. Dicks M, Romanelli F. Impact of novel active-learning approaches through iBooks and gamification in a reformatted pharmacy course. Am J Pharm Educ. 2019;83(3):6606.

25. Yunyongying P. Gamification: implications for curricular design. J Grad Med Educ. 2014;6(3):410-2.

26. Cook DA, Erwin PJ, Triola MM. Computerized virtual patients in health professions education: a systematic review and metaanalysis. Acad Med. 2010;85(10):1589-602.

27. Huang G, Reynolds R, Chandler C. Virtual patient simulation at U.S. and Canadian medical schools. Acad Med. 2007;82(5):446-51.

28. Posel N, Mcgee JB, Fleiszer DM. Twelve tips to support the development of clinical reasoning skills using virtual patient cases. Med Teach. 2015;37(9):813-8.

29. Bearman M. Is virtual the same as real? Medical students' experiences of a virtual patient. Acad Med. 2003;78(5):538-45.

30. Friedman CP, France CL, Drossman DA. A randomized comparison of alternative formats for clinical simulations. Med Decis Making. 1991;11(4):265-72.

31. Sobocan M, Turk N, Belon B. Paper problem-based learning (p-PBL) versus technology-enhanced decision-based PBL (d-PBL) in the classroom: is there an educational difference when using virtual patients? Med Sci Educ. 2017;27:119-22.

32. Benedict N, Schonder K, McGee J. Promotion of self-directed learning using virtual patient cases. Am J Pharm Educ. 2013;77(7):151.

33. Jackson JM, Skelton JA, Peters TR. Medical Students' Clinical Reasoning During a Simulated Viral Pandemic. Evidence of cognitive integration and insights on novices' approach to diagnostic reasoning. Med Sci Educ. 2020;30:767-74.

Publisher's Note Springer Nature remains neutral with regard to jurisdictional claims in published maps and institutional affiliations. 